\title{
Editorials
}

Donald Oxorn MD CM FRCPC

Gerald Edelist MD FRCPC

\section{Monitoring cardiac func- tion with transoesophageal echocardiography; esoterica or state of the art?}

of myocardial contraction. Assessment of most aspects of systolic function may also be undertaken. cardiac function in the operating room and intensive care unit are important thetists. In uncomplicated patients, assessment of heart rate, rhythm and blood pressure may suffice. More sophisticated monitoring techniques, however, may be required, depending on the patient's underlying cardiopulmonary reserve, the complexity of the operative procedure, and the physiological trespass resulting from therapeutic techniques such as positive pressure ventilation. The risk of invasive monitoring must always be balanced against the benefits of the clinical information accrued.

As progress has been made in the acquisition of cardiac images with transoesophageal echocardiography (TEE), the technique has moved from the confines of the cardiology laboratory into the operating room, the intensive care unit, and the emergency department. This has afforded anaesthetists the opportunity to become familiar with, and to develop expertise in its practice. Unlike transthoracic echocardiography, access to the patient's thorax and specific patient positioning are not required, thereby making TEE ideally suited to the patient population most commonly encountered by anaesthetists. Its use in patients with valvular heart disease, diseases of the aorta and pericardium, and congenital heart disease has recently been reviewed. ${ }^{1}$

By advancing the TEE probe into the stomach, a cross sectional view of the left ventricle may be obtained. At the level of the papillary muscles (midpapillary level), the territory of all three major coronary arteries are represented, and myocardial ischaemia may be detected by regional abnormalities

\section{Preload}

By tracing the endocardial border of the left ventricle at the midpapillary level, the cross sectional area of the left ventricular cavity is defined. When this measurement is made at end diastole, the end-diastolic area (EDA), a correlate of preload, is obtained. ${ }^{2,3}$ The major criticism of this method of assessing preload is that a measurement of area (two dimensions) is extrapolated to a measure of volume (three dimensions). Complicated formulae that incorporate a number of other dimensions in the calculation of left ventricular volume exist, but they are cumbersome, and may underestimate volume when measured angiographically. ${ }^{4}$ Automated border detection $(A B D)$ is a recent advance, in which the endocardial border is automatically recognized and traced, allowing serial changes in cardiac areas to be more rapidly delineated. ${ }^{5}$

\section{Contractility}

Measurement of LV contractility is difficult, because most parameters thought to reflect it are load sensitive; despite these limitations, ejection fraction has gained wide acceptance as a measure of LV contractility. When the EDA and end-systolic area (ESA) are both measured, the fractional area change (FAC) of the left ventricle may be obtained $(\% \mathrm{FAC}=100 \times[\mathrm{EDA}-\mathrm{ESA}] / \mathrm{EDA})$. This measurement made with either manual tracing of the endocardial border ${ }^{6,7}$ or $\mathrm{ABD},{ }^{8}$ has been found to correlate with radionuclide ejection fraction. 


\section{Afterload}

Left ventricular wall stress has been proposed as a measure of afterload ${ }^{9}$ but its measurement is cumbersome and not readily applicable to the management of patients in the operating room or intensive care unit.

\section{Cardiac Output}

The clinical gold standard in cardiac output determination is thermodilution with the use of a pulmonary artery catheter. Measurement of stroke volume may be accomplished by Doppler determination of the velocity of flow through one of several conduits, and multiplying it by the area of the conduit as measured by TEE; multiplying by heart rate yields cardiac output. The mitral $^{10}$ and aortic ${ }^{11}$ valve orifices, and the right, ${ }^{12}$ and left ${ }^{13}$ ventricular outflow tracts have been used as conduits. Problems include difficulty in positioning the probe to obtain adequate velocity measurements, compounded by the constant movement of the heart, the fact that the diameter of the conduit often changes during the cardiac cycle, and the need to leave the TEE probe in the patient for an extended period of time if multiple cardiac output determinations are required.

\section{Limitations of TEE as a Monitor of Systolic Function}

A major impetus to the use of TEE as a monitor of cardiac function is to avoid some of the pitfalls and problems of invasive monitoring, namely the complications of central line insertion and maintenance, and the sometimes dubious relationship between filling pressures and preload. The use of TEE in this regard, however, is not ideal. Training is required to achieve the necessary views and, even in expert hands, adequate views may not be obtainable. The constant moving of the heart with cardiac contraction and ventilator cycling may make the maintenance of these views difficult. Valvular regurgitation may introduce inaccuracies into the calculations. Most validating studies have been performed in patients with normal cardiac function and read offline; this may not reflect clinical reality. Most importantly, the technique may not be safe in critically ill patients whose tracheas are not intubated, or who cannot be assessed for contraindications to TEE.

\section{The Current Study}

In this issue of the Canadian Journal of Anaesthesia, Vedrinne and colleagues ${ }^{14}$ examine systolic blood pressure variation (SBPV) using arterial waveform analysis, and EDAi, ESAi (the $\mathrm{i}$ indicates the measurement is indexed to body weight), and FAC of the left ventricle with TEE, in patients with acute respiratory failure. Standard haemodynamic variables were undergoing positive pressure ventilation with measured pulmonary artery catheters. Changes in the above measurements as the I:E ratio was increased from $1: 3$ to $1: 1$ were also determined. The respiratory rate of the ventilator was adjusted so that six cardiac beats occurred during one respiratory cycle, with the initial beats occurring during inspiration, and the latter during expiration. The variations in EDAi, ESAi, and FAC of the left ventricle during the course of a complete respiratory cycle were analyzed.

The authors' results confirm findings of other investigators: left ventricular preload, as estimated by EDAi, decreased as the I:E ratio was increased, presumably because of an increase in mean airway pressure and diminished right ventricular output. ${ }^{15}$ During $1: 1$ ventilation, EDAi was maximal during lung inflation, and reached a nadir at the end of expiration. During lung inflation, diminished RV output would tend to decrease LV filling, but this is overshadowed by the increased LV filling from compression of the pulmonary veins. Systolic blood pressure variation during the course of a respiratory cycle is similarly explained. ${ }^{16}$

During 1:3 ventilation SBPV was present although less pronounced. This is not surprising, as it has been demonstrated previously that SBPV is more profound with coexistent hypovolaemia; ${ }^{17}$ relative hypovolaemia exists at an I:E ratio of $1: 1$ as is evident from the smaller EDAi. With 1:3 ventilation there was no variation of EDAi throughout the respiratory cycle, probably reflecting smaller changes in mean intrathoracic pressure at the smaller I:E ratio.

What conclusions can be drawn? Firstly, the importance of a clear description of methods in complicated experiments is extremely important and, unfortunately, this paper will have to be read several times to sort out the experimental protocol. How the patients were randomized, whether a crossover design was employed, and how the ventilation was adjusted is not clear. The results are not easily gleaned from the graphs and tables. One of the most important findings, the change in EDAi with inspiration and expiration during $1: 1$ ventilation, is only presented in the text. The authors refer to ejection fraction area which, by convention, should be called the fractional area of change (FAC). The conclusion that altering the I:E ratio may be used to determine volume status may be premature, as the magnitude of change in EDAi with a given level of intravascular volume has yet to be established.

However, the investigators have demonstrated the great potential of quantifying cardiac function with TEE, and applying the information in clinical investigation. The use of TEE in the diagnosis of life threatening haemodynamic disturbances has been firmly established, ${ }^{18}$ but its role in the day to day cardiovascular management of critically ill and surgical patients has yet to be defined. 


\section{Le monitorage cardiaque par échographie transoe- sophagienne : une norme ou une fantaisie ?}

Le monitorage et l'optimalisation de la fonction cardiaque en salle d'opération et aux soins intensifs constituent une fonction importante des anesthésistes. Chez les patients ordinaires, il suffit généralement d'évaluer la fréquence cardiaque et la pression artérielle. Des techniques de monitorage plus complexes sont quelquefois rendues nécessaires par l'état des réserves cardiopulmonaires, par la complexité des interventions et par l'agression physiologique de méthodes de traitement telles que la ventilation mécanique. Les risques du monitorage invasif doivent toujours être compensés par l'importance des renseignements qu'ils procurent.

D'abord confinée du laboratoire d'exploration cardiologique, l'échographie transoesophagienne(ÉTO), grâce à l'amélioration de la qualité des images cardiaques, s'est retrouvée en salle d'opération, à l'unité des soins intensifs et à la salle d'urgence. Cette migration a forcé les anesthésistes à se familiariser avec la technique et à acquérir de la compétence dans ce domaine. Contrairement à l'échocardiographie transthoracique, l'accès au thorax et l'adoption d'une position spéciale par le patient n'étant plus requis, l'ÉTO est devenue un outil de prédilection au service d'une population médicale qui intéresse l'anesthésiste au plus haut point. Une revue générale récente fait état de son utilisation chez des malades souffrant de valvulopathies, de maladies aortiques, péricardiques et congénitales. ${ }^{1}$

En avançant la sonde d'ÉTO vers l'estomac, on obtient une vue transversale du ventricule gauche. Au niveau des piliers (niveau papillaire moyen), on aperçoit les trois principales artères coronaires; les anomalies de la contraction myocardique permettent de déceler à ce niveau l'ischémie du myocarde. On peut aussi en grande partic évaluer la physionomie de la fonction systolique.

\section{La précharge}

Le tracé de la limite endocardique du ventricule gauche au niveau des piliers représente, en coupe transversale, la cavité ventriculaire gauche. Quand cette mesure est effectuée en fin de diastole, on obtient la surface télédiastolique (STD) du VG, le meilleur indice de la précharge. $^{2,3}$ On objecte toutefois que cette méthode d'évaluation n'est qu'une mesure de surface (mesure à deux dimensions) extrapolée à un volume (mesure à trois dimensions). Pour le calcul du volume VG, il existe des formules compliquées qui intègrent un certain nombre d'autres dimensions, mais elles sont lourdes, et sousestiment parfois le volume mesuré par angiographie ${ }^{4}$ Une acquisition récente, la détection automatisée du pourtour (Automated Border Detection: $\mathrm{ABD}$ ) qui reconnaît automatiquement et trace la limite endocardique permet de détecter plus facilement les changements en succession. ${ }^{5}$

\section{La contractilité}

La contractilité VG est difficile à mesurer parce que tous les paramètres supposés la refléter sont sensibles aux conditions de charge ; malgré ces limitations, la fraction d'éjection est généralement acceptée comme une mesure de la contractilité VG. Quand la STD et la surface télésystolique (STS) sont toutes les deux mesurées, la fraction de raccourcissement de surface (FRS) du ventricule gauche peut être calculée (\% FRS $=100 \times$ [STD - STS] STD). Cette mesure a une bonne corrélation avec la fraction d'éjection déterminée en radioangiographie par tracé manuel du bord endocardique ${ }^{6,7}$ ou par ABD.

\section{La postcharge}

La contrainte systolique VG a été proposée comme mesure de la postcharge ${ }^{9}$ mais cette mesure est compliquée et difficilement applicable dans le contexte de la salle d'opération ou de l'unité de soins intensifs.

\section{Le débit cardiaque}

La méthode de thermodilution à l'aide d'un cathéter artériel pulmonaire représente le standard reconnu de la mesure du débit cardiaque. Toutefois, celui-ci peut aussi être évalué par l'estimation à travers un des conduits, au Doppler, de la vélocité du débit, multipliée par la surface de ce conduit mesurée par ÉTO. Les conduits utilisés sont l'orifice mitral ${ }^{10}$ et aortique, ${ }^{11}$ la chambre de chasse du ventricule droit ${ }^{12}$ et gauche. ${ }^{13}$ Parmi les problèmes propres à cette méthode, mentionnons la difficulté de bien placer la sonde pour obtenir des mesures convenables de la vélocité, la sonde étant constamment ballottée par les mouvements cardiaques; le fait que le diamètre du conduit change fréquemment pendant les phases du cycle cardiaque et, finalement, la nécessité de laisser la sonde d'ÉTO en place pour de longues périodes si on doit mesurer le débit cardiaque à répétitions. 


\section{Les limites de l'ÉTO comme moniteur de la fonction systolique}

L'utilisation de l'ÉTO comme moniteur de la fonction cardiaque est largement motivée par le fait qu'elle permet d'éviter les embûches et les problèmes causés par le monitorage invasif, et, plus spécifiquement, les complications dues à l'insertion et au maintien de cathéters centraux; et aussi par la corrélation contestable entre les pressions de remplissage et la précharge. Sous cet aspect, l'usage de l'ÉTO n'est pas idéal non plus. L'acquisition de vues valables nécessite une formation et même, entre les mains d'experts, ces vues ne sont pas toujours adéquates. Il est aussi difficile de les conserver à cause du déplacement du coeur à chaque contraction et avec le cycle du ventilateur. $\mathrm{La}$ régurgitation valvulaire peut aussi fausser les calculs. La plupart des études de validation ont été réalisées sur des coeurs normaux et interprétées en différé, ce qui contraste avec la réalité clinique. Il est encore plus important de noter que la technique est dangereuse chez des grands malades dont la trachée n'est pas intubée ou chez qui les contre-indications à l'ÉTO ne peuvent être évaluées.

\section{L'étude présente}

Dans ce numéro du Journal canadien d'anesthésie, Vedrinne et $a l .1{ }^{14}$ ont étudié la variation de la pression systolique (VPAS) sous ventilation en volume contrôlé en utilisant l'analyse de forme d'onde, la STDi, la STSi («i» pour indexation au poids corporel) et la FRS du ventricule gauche, les mesures étant effectuées pendant l'ÉTO chez des insuffisants respiratoires aigus. De plus, les variables hémodynamiques standards étaient déterminées avec un cathéter artériel pulmonaire. Les changements des mesures étaient déterminés pendant que le rapport I:E augmentait de $1: 3$ à 1:1. En outre, la fréquence du ventilateur était réglée de façon à ce que six battements cardiaques surviennent pendant un cycle respiratoire, les premiers battements arrivant pendant l'inspiration et les derniers pendant l'expiration. Les variations de la STDi, STSi et de la FRS du ventricule gauche étaient analysés pendant un cycle respiratoire complet.

Les résultats obtenus par les auteurs de cet article confirment ceux qu'ont rapportés d'autres chercheurs : la précharge VG telle qu'évaluée par la STDi diminue à mesure que la rapport I:E augmente, parce que la pression moyenne des voies aériennes augmente et que conséquemment le débit du ventricule droit diminue. ${ }^{15}$ Pendant la ventilation $1: 1$, la STDi était maximale pendant l'insufflation pulmonaire et atteignait son nadir à la fin de l'expiration. Pendant l'insufflation pulmonaire, la chute du débit
VD aurait tendance à réduire le remplissage $V G$, mais cet effet est annulé par l'augmentation du remplissage VG due à la compression des veines pulmonaires. $\mathrm{La}$ variation de la pression artérielle systolique au cours d'un cycle respiratoire s'explique de la même façon. ${ }^{16}$

Pendant la ventilation $1: 3$, la VPAS variait aussi, mais moins nettement. Ceci n'a rien de surprenant parce qu'il a déjà été démontré que la VPAS est plus profonde pendant l'état d'hypovolémie $;^{17}$ comme le montre la baisse accentuée de la STDi, il existe une hypovolémie relative lorsque le rapport I:E est de $1: 1$. Pendant le cycle respiratoire, la STDi ne variait pas, ce qui reflétait vraisemblablement des changements moins importants de la pression intrathoracique moyenne lorsque le rapport I:E est plus bas.

Que pouvons-nous conclure ? Dans des expériences aussi compliquées, il est très important que la description des méthodes soit claire, mais malheureusement, il faut lire cet article plusieurs fois avant d'en comprendre le protocole expérimental. On n'y voit pas clairement de quelle façon la randomisation a été effectuée, si c'était une étude avec croisement et comment on avait réglé la ventilation. Les résultats sont difficilement extraits des graphiques et des tableaux. Une des constatations les plus importantes, la variation de la SDTi pendant l'inspiration et l'expiration en ventilation 1:1 n'apparaît que dans le texte. Les auteurs réferent à la surface de la fraction d'éjection qui, par convention, devrait s'appeler la fraction de raccourcissement systolique (FRS). Il semble prématuré de conclure que l'altération du rapport I:E puisse servir à déterminer l'état de la volémie étant donné que l'ordre de grandeur du changement de la STDi pour un niveau de volume intravasculaire donné n'est pas encore établi.

Cependant, ces chercheurs ont démontré les grandes possibilités offertes par l'évaluation quantitative de la fonction avec l'ÉTO et par son application à la recherche clinique. L'urilisation de l'ÉTO pour le diagnostic des perturbations hémodynamiques vitales a été fermement établie ${ }^{18}$ mais son rôle pour la gestion cardiovasculaire des grands malades et des opérés n'est pas encore défini.

\section{References}

1 Oxorn D, Edelist G, Stafford Smith M. An introduction to transoesophageal echocardiography: II. Clinical applications. Can J Anaesth 1996; 43: 278-94.

2 Swenson JD, Harkin C, Pace NL, Astle K, Bailey P. Transesophageal echocardiography: an objective tool in defining maximum ventricular response to intravenous fluid therapy. Anesth Analg 1996; 83: 1149-53.

3 Cheung AT, Savino JS, Weiss SJ, Aukburg SJ, Berlin JA. Echocardiographic and hemodynamic indexes of left 
ventricular preload in patients with normal and abnormal ventricular function. Anesthesiology 1994; 81 : 376-87.

4 Smith MD, MacPhail B, Harrison MR, Lenhoff $S L$, DeMaria $A N$. Value and limitations of transesophageal echocardiography in determination of left ventricular volumes and ejection fraction. J Am Coll Cardiol $1992 ; 19: 1213-22$.

5 Perrino AC Jr, Luther MA, O'Connor TZ, Coben IS. Automated echocardiographic analysis. Examination of serial intraoperative measurements. Anesthesiology 1995; 83: 285-92.

6 Clements FM, Harpole DH, Quill T, Jones $R H$, $M c C a n n R L$. Estimation of left ventricular volume and ejection fraction by two dimensional transoesophageal echocardiography: comparison of short axis imaging and simultaneous radionuclide angiography. $\mathrm{Br} \mathrm{J}$ Anaesth 1990; 64: 331-6.

7 Samuelsson S, Brodin LA, Broman $M$, Öwall $A$, Settergren $G$. Comparison between transesophageal Doppler echocardiography and nuclear cardioangiography for the evaluation of left ventricular filling during coronary artery bypass grafting. Anesth Analg 1995; 80: 41-6.

8 Liu $N$, Darmon P-L, Saada $M$, et al. Comparison between radionuclide ejection fraction and fractional area changes derived from transesophageal echocardiography using automated border detection. Anesthesiology 1996; 85: 468-74.

9 Reichek N, Wilson J, St. Jobn Sutton M, Plappert TA, Goldberg $S$, Hirshfeld $J W$. Noninvasive determination of left ventricular end-systolic wall stress: validation of the method and initial application. Circulation 1982; 65: 99-108.

$10 P u M$, Griffin BP, Vandervoort PM, Leung $D Y$, Cosgrove DM III, Thomas JD. Intraoperative validation of mitral inflow determination by transesophageal echocardiography: comparison of single-plane, biplane, and thermodilution techniques. J Am Coll Cardiol 1995; 26: 1047-53.

11 Darmon PL, Hillel Z, Mogtader A, Mindich B, Thys D. Cardiac output by transesophageal echocardiography using continuous-wave Doppler across the aortic valve. Anesthesiology 1994; 80: 796-805.

12 Maslow A, Comunale ME, Haering JM, Watkins J. Pulsed wave Doppler measurement of cardiac output fron the right ventricular outflow tract. Anesth Analg 1996; 83: 466-71.

13 Feinberg MS, Hopkins WE, Davila-Roman VG, Barzilai $B$. Multiplane transesophageal echocardiographic Doppler imaging acurately determines cardiac output measurement in critically ill patients. Chest 1995; 107 : 769-73.
14 Vedrinne J-M, Duperret S, Decaillot $F$, Gratadour $P$, Motin J. Haemodynamic changes induced by two I:E ratios; a transoesophageal echocardiographic study. Can J Anaesth 1997; 44: 354-359.

15 Jardin F, Delorme $G$, Hardy $A$, Anvert B, Beauchet $A$, Bourdarias $J P$. Reevaluation of hemodynamic consequences of positive pressure ventilation: emphasis on cyclic right ventricular afterloading by mechanical lung inflation. Anesthesiology 1990; 72: 966-70.

16 Jardin F, Farcot JC, Gueret P, Prost JF, Ozier $\Upsilon$, Bourdarias JP. Cyclic changes in arterial pulse during respiratory support. Circulation $1983 ; 68: 266-74$.

17 Coriat $P$, Vrillon $M$, Perel $A$, et al. A comparison of systolic blood pressure variations and echocardiographic estimates of end-diastolic left ventricular size in patients after aortic surgery. Anesth Analg 1994; 78: 46-53.

18 Heidenreich PA, Stainback RF, Redberg RF, Schiller NB, Coben NH, Foster E. Transesophageal echocardiography predicts mortality in critically ill patients with unexplained hypotension. J Am Coll Cardiol 1995; 26: 152-8. 